\title{
Designing and implementing effective venous thromboembolism prevention protocols: lessons from collaborative efforts
}

\author{
Greg Maynard · Jason Stein
}

Published online: 10 November 2009

(C) The Author(s) 2009. This article is published with open access at Springerlink.com

\begin{abstract}
Hospital acquired venous thromboembolism (VTE) is a major source of morbidity and mortality, yet proven prevention measures are often underutilized. The lack of a validated VTE risk assessment model, difficulty integrating VTE risk assessment and prevention protocols into the routine process of care, and the lack of standardized metrics for VTE prophylaxis have all been barriers. Recently, a VTE risk assessment/prevention protocol has been validated, leading to portable strategies achieving breakthrough levels of adequate prophylaxis in a variety of inpatient settings. VTE prevention protocol design and implementation strategies have been collected in implementation guides available from the Society of Hospital Medicine and the Agency for Healthcare Research and Quality. These guides were the centerpieces of national collaborative efforts to improve VTE involving over 150 medical centers, honing the approach to accelerate improvement described in this article. Embedding a VTE prevention protocol into admission, transfer, and perioperative order sets is a key strategy. A VTE prevention protocol is defined as a VTE risk assessment with no more than three levels of risk, tightly linked to recommended prophylaxis for each level. A balance between the need to provide protocol guidance and the need for efficiency and ease-of-use by the clinician must be maintained. The power of this protocol driven approach is bolstered by a quality improvement framework, multidisciplinary teams, ongoing
\end{abstract}

G. Maynard $(\bowtie)$

Division of Hospital Medicine, Department of Medicine, University of California, San Diego, San Diego, CA 921038485, USA

e-mail: gmaynard@ucsd.edu

J. Stein

Emory University, Atlanta, GA, USA monitoring of the process, and real time identification and mitigation of non-adherents via a technique that measures progress and prompts concurrent intervention, an approach we call "measure-vention."

Keywords Deep vein thrombosis .

Venous thromboembolism - Pulmonary embolism .

Prevention · Protocols · Standardized order sets .

Measure-vention

\section{Introduction}

Pulmonary embolism (PE) and deep vein thrombosis (DVT), collectively referred to as venous thromboembolism (VTE), represent a major public health problem, affecting hundreds of thousands of Americans each year [1]. At least 100,000 deaths, and perhaps over 200,000 deaths, are attributable to VTE each year in the United States alone [1]. VTE is primarily a problem of hospitalized and recently hospitalized patients [2, 3], and PE is frequently estimated to be the most common preventable cause of hospital death [4-6].

Pharmacologic methods to prevent VTE are safe, effective, cost-effective, and advocated by authoritative guidelines [7]. Even though the majority of medical and surgical inpatients have multiple risk factors for VTE, large prospective studies continue to demonstrate that these preventive methods are significantly underutilized, often with only $30-50 \%$ eligible patients receiving prophylaxis [8-12].

Recent investigations like (ENDORSE) [12] offer a perspective of VTE prevention performance from around the world. This cross sectional survey encompassed 358 medical centers from 32 countries. Only $58.5 \%$ of surgical 
patients and $39.5 \%$ of medical patients were on prophylaxis consistent with ACCP [7] guidelines, and the United States performance was only marginally better than the world mean, with less than $50 \%$ of medical patients receiving guideline recommended prophylaxis.

The toll in preventable mortality and morbidity has not gone unnoticed by those in health advocacy groups, policy makers, and payers. The National Quality Forum has already endorsed that each patient be evaluated for their risk of VTE on admission and regularly thereafter [13], and The Joint Commission is moving towards standards that will hold medical centers accountable for ensuring that patients will have VTE prophylaxis in place within $24 \mathrm{~h}$ of hospital admission, and within $24 \mathrm{~h}$ of transfer to critical care settings, or demonstrate a risk assessment or contraindications to justify why it is not in place [14]. Furthermore, the Centers for Medicare and Medicaid Services (CMS) recently ruled that if a patient develops VTE during hospitalization for total knee or hip replacement, the hospital will not be paid for the added expense of that complication [15].

Multiple reasons have been invoked to explain this persistent under-utilization, in spite of good faith efforts in medical centers to improve performance, and ever increasing external pressures. These include a lack of physician familiarity or agreement with guidelines, underestimation of VTE risk, concern over risk of bleeding, and the perception that the guidelines are resource intensive or difficult to implement in a practical fashion [16]. While many VTE risk assessment models are available in the literature [17-21], the absence until just recently of prospectively validated models, and issues regarding ease of use have hampered widespread integration of VTE risk assessments into order sets and inpatient practice.

\section{Successful local and national VTE prevention efforts}

Recognizing the importance of improving VTE prophylaxis, we initiated an Agency for Healthcare Research and Quality (AHRQ) funded project in 2005 to optimize prevention of hospital-acquired VTE in our 350 bed tertiary care center, using techniques that could be replicated in a variety of other institutions. A full description of techniques used and the results obtained is in press [22] and will be available elsewhere, but a few highlights are instructive. We designed, piloted, and implemented a VTE prevention protocol for all adult medical surgical patients, via integration of a simple VTE risk assessment model into admission and transfer order sets. Each level of VTE Risk was firmly linked to a menu of acceptable prophylaxis options, and we augmented the performance of the protocol with methods such as audit and feedback, education, and real-time identification and mitigation of non-adherents to the protocol.

For the first time, a VTE risk assessment model/prevention protocol was validated in a number of important ways. Ease of use and inter-observer agreement for VTE risk level and a judgment of "adequate" prophylaxis were very high (kappa score 0.81 and 0.90 , respectively), and the VTE risk assessment model was predictive of VTE risk. The percent of patients on adequate prophylaxis improved each year, from $58 \%$ of sampled inpatients to over $98 \%$ of inpatients sampled in the latter half of 2007 and all of 2008. Significant reductions for the risk of HA VTE [RR 0.69 (0.47-0.79)] and preventable HA VTE [RR 0.14 (0.06$0.31)$ ] occurred, and there was no detectable increase in heparin induced thrombocytopenia or prophylaxis-related bleeding as assessed by chart review of administrative data.

We have published implementation guides now available in both web and stand alone formats [23-25]. These guides, drawing from our local experience as well as other experts and improvement team leaders, take improvement teams in a step-by-step fashion through the process of effective VTE protocol design, implementation, and monitoring. The principles outlined in our implementation guides have served as a road map for a number of successful mentored implementation collaborative efforts administered via the Society of Hospital Medicine (SHM), AHRQ, and more recently the Institute for Healthcare Improvement (IHI). The advice and principles outlined below have been tested and found to be effective and practical in over 150 medical centers of all types (academic and community, small and large, rural and urban, paper and electronic order environments).

\section{Overview of infrastructure: basic ingredients for effective efforts}

To implement effective protocols minimizing incidence of hospital-acquired VTE, while at the same time minimizing adverse outcomes, redesign is needed in both care delivery and performance tracking. Essential elements to reach breakthrough levels of improvement include:

- Institutional support and prioritization for the initiative, expressed in terms of a commitment to standardize the process of providing VTE prophylaxis, and reasonable support to facilitate implementation and monitoring of results.

- A multidisciplinary team or steering committee focused on reaching VTE prophylaxis targets and reporting to key medical staff committees. Physician leadership is a necessary component of the team. High volume providers such as hospitalists and critical care 
physicians, or prominent surgery leaders, are desirable to have as leaders for the improvement effort.

- Specific goals or aims which are ambitious, timedefined, and measurable.

- Protocols that standardize VTE risk assessment and prophylaxis and embed this process in the flow of normal patient care.

- Reliable data collection and performance tracking (suggested metrics described in more detail below), with education and refinement of the protocol driven by the ongoing observation and measurement process.

As in all improvement efforts, we endorse piloting small tests of change, and careful vetting of VTE prevention order sets before attempting wide implementation.

\section{The essential intervention: a VTE prevention protocol}

We define a VTE prevention protocol to be a standardized VTE risk assessment, linked to a menu of appropriate VTE prophylaxis options for each level of risk [23-25]. Guidance for management of patients with contraindications to pharmacologic prophylaxis should also be included. The best protocols provide decision support at the point of care, and yet are user friendly and efficient to use, and preserve the ability to customize care for special patient situations or circumstances [23-26].

Who administers the protocol?

Conceivably, anyone in the medical center (e.g. a nurse or pharmacist) could administer the risk assessment model and present the results to the physician for action. However, our collective experience suggests that the VTE prevention protocol is most effective when embedded in commonly used admission, transfer, and perioperative order sets. Across VTE collaboratives, the physician consistently appears to be in the best position to understand all components of VTE risk, along with the possible contraindications to pharmacologic prophylaxis. Furthermore, an immediate connection in time and space of the VTE risk assessment to the ordering process makes for a more reliable and direct route for ordering adequate prophylaxis.

How should I design the risk assessment into the order set?

We recommend integrating a simple text based model with no more than three levels of VTE risk. An illustrative paper order set is depicted in the Appendix. While this example depicts enoxaparin as the low molecular weight heparin of choice, local formulary issues and medical staff opinion will dictate these choices, and with this illustration we are not implying one is superior to the other.

At one extreme of VTE risk are patients that would benefit from both a low molecular weight heparin or fondaparinux and mechanical prophylaxis (while not depicted in this example, some institutions also offer warfarin as an option for these high risk patients). Major orthopedic surgery, multiple major trauma, spinal cord injury with paresis, and abdominal/pelvic cancer patients undergoing surgery are common choices for patients in this very high risk category. Choices for renal insufficiency and end stage renal disease are offered with simple caveats in parentheses. In our medical center, 15-20\% of our inpatients fall into this highest risk category.

At the other extreme are patients at such low risk for VTE they do not require any prophylaxis other than education and ambulation. This model accepts a paradigm in which almost all inpatients are at risk for VTE and need pharmacologic prophylaxis, with the exception of the few who are expected to be in the medical center for less than $48 \mathrm{~h}$, or who have a paucity of risk factors and are fully and independently ambulating. This low risk subset represents less than $5 \%$ of inpatients at our medical center.

The majority of inpatients occupy the middle category of VTE risk. While there is some evidence favoring low molecular weight heparin (LMWH) versus unfractionated heparin (UFH) in subsets of this population [27, 28], evidence is fairly weak at this risk level, and either choice is usually seen as acceptable. Note that UFH 5000 units dosed every $12 \mathrm{~h}$ has been relegated a secondary role, suitable only for underweight or geriatric patients. This choice is admittedly somewhat arbitrary, but the trials comparing LMWH to UFH regimens used 5000 units q $8 \mathrm{~h}$ dosing schedules in the comparison arm [27-30]. On the whole, there is more evidence for using UFH $5000 \mathrm{q} 8 \mathrm{~h}$ schedules compared to UFH $5000 \mathrm{q} 12 \mathrm{~h}$ regimens. Attempts to tease out populations in which UFH $5000 \mathrm{q} 8 \mathrm{~h}$ is acceptable but UFH $5000 \mathrm{q} 12 \mathrm{~h}$ regimens are not are generally not worth the effort, and lead to unnecessary clutter and complexity to order sets.

Contraindications and a full listing of VTE risk factors should not occupy precious "real estate" on the front page of an order set, but should be readily accessible by instructions embedded there. Sequential compression devices are specifically listed as the default choice for mechanical prophylaxis. Mechanical prophylaxis is relegated to an adjunctive role, or as a first line choice when pharmacologic prophylaxis is not feasible. Aspirin is not listed as an acceptable choice for DVT prophylaxis, which is consistent with prominent guidelines [7]. Improvement teams need not feel restricted in the design details of the order set or the choices they offer for each level of risk, 
providing they retain the most important design concepts and avoid the most common errors outlined below.

Common errors in constructing/implementing a VTE prevention order set

Not providing enough guidance: a prompt is not a protocol

Many centers have order sets that list options for VTE prophylaxis without providing any guidance for which choice is most appropriate or desirable. Mechanical prophylaxis, varying pharmacologic agents with different doses, and no prophylaxis are inappropriately offered as equal options, even though most inpatients have significant VTE risk factors [7, 11], and in spite of strong evidence based recommendations [7] relegating mechanical prophylaxis to an adjunctive role for pharmacologic prophylaxis (unless there are contraindications to pharmacologic prophylaxis).

Providing too much guidance: order sets can become too complicated

It is tempting to create an order set that provides comprehensive guidance and outlines the best prophylaxis for the entire spectrum of conditions. Improvement teams must strike a fine balance between providing a good risk assessment for the great majority of the inpatient population, yet keeping things simple and efficient in everyday use. We have encountered three page stand-alone order VTE prophylaxis order sets that would provide excellent guidance if used, but they simply collect dust and are adapted by only a minority of providers. Some order sets offer 4-6 levels of VTE risk, but the evidence to distinguish the levels of risk, and the differences in attendant prophylaxis choices, is often weak. We have found that 2-3 levels of VTE risk are enough.

Many centers have tried to adapt point based models inspired by the pioneering work of Caprini and colleagues $[18,21]$. In theory, this model is attractive. Risk factors depicting the clinical setting and the patient are assigned points, and the cumulative point total is entered into the order set, with guidance for prophylaxis hinging on this cumulative point total. In practice, this risk assessment method is often fraught with problems. Hurried clinicians do not reliably add up the points for each risk, and interobserver agreement suffers as a result. The point scoring system is somewhat arbitrary, and has not yet been validated in the literature. Most importantly, the point based systems are too long and bulky to integrate into a variety of existing order sets, and as a result, reliability of use and wide adaption is problematic.
Offering non-pharmacologic prophylaxis as a first line option

Pharmacologic prophylaxis is recommended as the first line choice for inpatients at risk for VTE. Mechanical prophylaxis can (and often should) be offered as an adjunct to pharmacologic prophylaxis, but it should not be offered as a first line choice for prophylaxis in the absence of contraindications to VTE prophylaxis.

\section{Link between risk level and prophylaxis choices are separated in time or space}

Ideally, the VTE risk assessment is performed quickly, and the choices for appropriate prophylaxis are directly and inescapably linked to each level of risk. If the act of ordering VTE prophylaxis is separated in any way from the risk assessment, the reliability of ordering adequate prophylaxis deteriorates.

\section{Failure to revise pre-existing and conflicting order sets}

Most institutions have a variety of admission, transfer, and order sets in place. These order sets often already "touch" the majority of adult inpatients, and they often have a highly variable approach to VTE prophylaxis embedded within them. We strongly urge improvement teams to examine all existing admission, transfer, and perioperative order sets with reference to VTE prophylaxis. The designers and users of these order sets should be approached to build consensus on using the redesigned, standardized, protocol-driven VTE prophylaxis orders, with the intent to strip out all non-standardized VTE prevention orders, and replace them with the new, standardized version.

\section{Modular versus stand alone orders}

If the order set is constructed properly, it can often be designed as a VTE prevention "module," making it easier to integrate into the pre-existing order sets referenced above. This is preferable to order sets designed to stand alone, but even stand alone orders can be integrated into the flow of admissions and transfers with a little ingenuity. Clipping the VTE order set on to "History and Physical" forms or onto pre-existing order sets may increase adaption to an acceptable level, though we prefer modular order set design whenever possible.

\section{Enhancing the power of the protocol}

Skillful introduction of a good order set that reaches most patients has often yielded observed VTE prophylaxis rates 
of $70-85 \%$ (from baseline performance of $35-55 \%$ ) in our local experience and in collaborative VTE prevention efforts. This performance boost is a great accomplishment, but such an approach alone is not sufficient. No matter how clear and concise the order set is, it will not always be used correctly. Moreover, acutely ill patients often have transient changes in the risk/benefit ratio of using anticoagulation, leading to lapses in VTE prophylaxis that persist beyond transient bleeding risk. To reach higher levels of performance under these constraints, a multifaceted approach using a variety of techniques has been effective in the literature [16, 22-25, 31-36] and in the collaborative. Educational programs alone [32, 37, 38] are not generally sufficient to bring about reliable VTE prophylaxis, but are needed to foster appropriate use of order sets and protocols. Periodic audit and feedback and computerized decision support can also be very effective [31, 39-43], particularly when there is an institutional protocol to hold up as the defining standard for adequate prophylaxis, but some of the more sophisticated tools are beyond the reach of many improvement teams.

\section{Situational awareness and measure-vention}

One method to enhance the power of the VTE prevention protocol has distinguished itself from all the others. This method, which we call "measure-vention," involves identifying and measuring patients on potentially inadequate prophylaxis and intervening on them in real time. This strategy has proven successful in a variety of environments
[22, 33]. The measure-vention technique for VTE prophylaxis most commonly follows a series of steps as described below.

The medication administration record (MAR) or an automated report (often generated by pharmacy) is presented to front line staff, identifying the VTE prophylaxis status of each patient on the ward. Advanced versions of this approach have actually classified each patient on the ward as being "green" (an order in place for therapeutic or prophylactic anticoagulation), the "yellow" (mechanical prophylaxis without pharmacologic prophylaxis), or "red" (no VTE prophylaxis orderd), see Fig. 1. Extracting this information from the MAR into a report creates a situational awareness, calling for explicit action on the part of the front line staff member.

The staff member (usually a staff nurse or charge nurse, but sometimes a pharmacist) is then called upon to intervene on patients that seem to be non-adherent to the protocol. For example, if a patient is classified as red, nursing can be authorized to place sequential compression devices on the patient - and if there are no obvious bleeding problems and any VTE risk factor is present - can place a simple templated note on the chart and text page the physician, asking them to either place the patient on pharmacologic prophylaxis or state the reason they choose not to do this. This method can quickly bolster VTE prophylaxis rates to 95\% [33], and fatigue from alerts can be minimized if the intervention part of the strategy is deployed after the order set is launched. The medical staff leadership and administration need to make sure the front line staff are comfortable carrying out this
Fig. 1 The measure-vention strategy depicted in this figure is a real-time dashboard formatted to classify patient's VTE prophylaxis status. In actual use and as depicted in the online version of this article, each patient's status is categorized as red (no VTE prophylaxis ordered), yellow (mechanical prophylaxis only, with no pharmacologic prophylaxis), and green (pharmacologic or therapeutic anticoagulation)

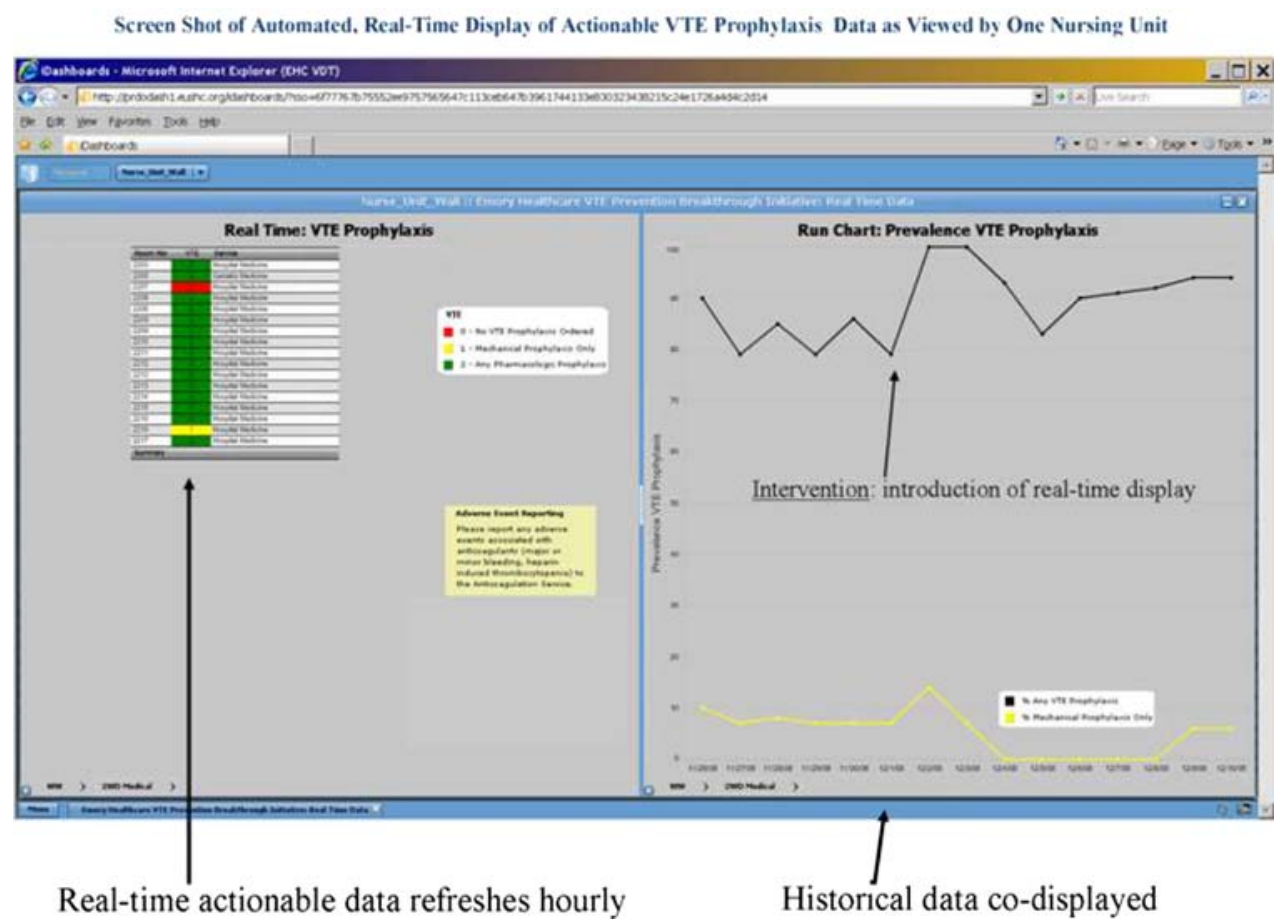


strategy, and make it clear that "shooting the messenger" is unacceptable.

Measure-vention as described above provides the means to establish reliable, easy to understand metrics for VTE prophylaxis. The percent of patients on anticoagulation based prophylaxis, and the percent of patients on any prophylaxis, while not perfect metrics, are easy to assess and relatively easy to automate. These measures are actionable on the front line, yet are suitable for tracking, trending, and creating roll-up reports that could be reported to key medical staff committees and governing boards.

\section{Dueling guidelines and other barriers}

In many centers, implementing a standardized VTE prevention protocol meets with resistance from one or more groups of physicians, and more often than not, the objecting physicians are orthopedists. While many orthopedists are very comfortable with pharmacologic prophylaxis and will have no problem with this approach, we estimate that about one third of centers run into stiff resistance from their orthopedic physicians. This resistance gained some momentum when the American Academy of Orthopedic Surgeons (AAOS) disseminated a new guideline [44] that differs in many ways from the most prominent guideline [7] from the American College of Chest Physicians (ACCP). The AAOS guidelines, in a point of distinction from the ACCP guidelines, allow the use of aspirin as a prophylactic agent, in patients at high risk of bleeding, and in patients at standard risk of bleed and standard risk of PE with joint replacement. The differences between the two guidelines are largely explained by the viewpoint of the authors and the methods they used for their supporting literature review. The AAOS guidelines take the view that the literature underestimates the bleeding risk of pharmacologic prophylaxis with joint replacement, and that bleeding into the wound or joint can cause long term joint problems. They also focused only on symptomatic PE, discounting the morbidity caused by symptomatic DVT, and discounting the relationship between asymptomatic DVT found on routine screening and the development of symptomatic problems.

Improvement teams faced with the resistant physician group (be they orthopedic group or others) can not let this resistance stop their efforts to improve VTE prophylaxis for the larger population, but a pitched battle and mandating adherence to one standardized protocol is generally counterproductive in this setting, especially in view of the conflicting guidance from these two guidelines. Orthopedic groups who feel strongly that they should follow AAOS guidelines instead of ACCP guidelines should be "carved out" of the standardization used for the rest of the adult medical/surgical population, though they should be expected to standardize their care within the confines of the alternate guideline.

There are also guidelines from other sources concerning VTE prevention $[45,46]$ covering special populations like oncology patients and obstetrics/gynecology patients. These guidelines are largely congruent with ACCP guidelines, but improvement teams should consider closely if there are other services that should be allowed "carve out" status.

\section{Summary}

Hospital acquired VTE related morbidity and mortality are huge public health problems, and improving performance on VTE prevention is a moral and public health imperative. Lessons gleaned from local success stories and national collaborative efforts can accelerate improvement in this vital area. Integrating a simple VTE risk assessment into VTE prevention orders that are positioned to capture the majority of admissions and transfers is an essential strategy. The power of this protocol driven approach can be bolstered by using a quality improvement framework, a multidisciplinary team approach, ongoing monitoring of the process, and real time identification and mitigation of non-adherents via the measure-vention technique.

Acknowledgements Implementation guides and the VTE Prevention Resource Room are based on the work other great collaborators and staff at the Society of Hospital Medicine. This review refers to an improvement and research project that was originally funded under grant number Grant No. 1U18HS015826-01 from the Agency for Healthcare Research and Quality, U.S. Department of Health and Human Services. We remain grateful for their support.

Open Access This article is distributed under the terms of the Creative Commons Attribution Noncommercial License which permits any noncommercial use, distribution, and reproduction in any medium, provided the original author(s) and source are credited. 


\section{Appendix}

Venous Thromboembolism Risk Assessment \& Prophylaxis PHYSICIAN ORDER Sheet Complete Assessment at ADMISSION, POST-OP, AND TRANSFER

\begin{tabular}{|c|c|}
\hline \multicolumn{2}{|c|}{ DVT/ PE RISK LEVEL \& PROPHYLAXIS ORDERS } \\
\hline \multirow{3}{*}{$\begin{array}{l}\qquad \square \text { LOW Risk } \\
\text { Observation patients, expected LOS }<48 \text { hrs: Minor/ } \\
\text { Ambulatory surgery or Age }<50 \text { and NO other risk } \\
\text { factors , or Already on therapeutic anticoagulation }\end{array}$} & $\square$ Early ambulation, education \\
\hline & $\square$ Education \\
\hline & \\
\hline \multirow[t]{2}{*}{$\begin{array}{l}\qquad \square \text { Moderate Risk } \\
\text { Most medical /surgical patients } \\
\text { CHF,pneumonia, active inflammation, advanced } \\
\text { age, dehydration, varicose veins, less than fully and } \\
\text { independently ambulatory, many other factors. All } \\
\text { patients not in the Low or Highest Risk Categories } \\
\text { (see reverse for more risk factors) }\end{array}$} & $\begin{array}{l}\text { CHOOSE ONE PHARMACOLOGIC option } \\
\square \text { Enoxaparin } 40 \mathrm{mg} \mathrm{SC} \mathrm{q} 24 \mathrm{hrs} \\
\square \text { Enoxaparin } 30 \mathrm{mg} \mathrm{SC} \mathrm{q} 24 \mathrm{hrs} \quad \text { (renal insufficiency dosing) } \\
\square \text { Heparin } 5000 \text { units SC q } 8 \mathrm{hrs} \\
\square \text { Heparin } 5000 \text { units SC every } 12 \mathrm{hrs} \text { (if weight }<50 \mathrm{~kg} \text { or age }>75 \text { ) }\end{array}$ \\
\hline & $\begin{array}{l}\text { Also (OPTIONAL) } \\
\square \text { Sequential compression device }\end{array}$ \\
\hline \multirow[t]{2}{*}{$\begin{array}{l}\qquad \square \text { Highest Risk } \\
\text { Elective hip or knee arthroplasty } \\
\text { Acute spinal cord injury with paresis } \\
\text { Multiple major trauma } \\
\text { Abdominal or pelvic surgery for cancer }\end{array}$} & $\begin{array}{l}\text { CHOOSE ONE PHARMACOLOGIC option } \\
\square \text { Enoxaparin } 40 \mathrm{mg} \mathrm{SC} \mathrm{q} \mathrm{day} \\
\square \text { Enoxaparin } 30 \mathrm{mg} \mathrm{SC} \mathrm{q} 24 \mathrm{hrs} \text { (for renal insufficiency) } \\
\square \text { Heparin } 5000 \text { units SC q } 8 \mathrm{hrs} \text { (End stage renal disease only) } \\
\square \text { Enoxaparin } 30 \mathrm{mg} \mathrm{SC} \mathrm{q} 12 \mathrm{hrs} \text { (knee replacement) } \\
\square \text { Fondaparinux } 2.5 \mathrm{mg} \mathrm{SC} \text { q day }\end{array}$ \\
\hline & - Sequential compression device \\
\hline \multicolumn{2}{|c|}{$\begin{array}{l}\text { OR } \\
\text { The risk of adverse effects of pharmacologic prophylaxis outweighs the risk of DVT / PE } \\
\text { Contraindication to pharmacologic prophylaxis (see reverse): } \\
\square \text { Mechanical prophylaxis with sequential compression device OR } \\
\square \text { Contraindicated (peripheral vascular disease or wounds) }\end{array}$} \\
\hline
\end{tabular}

SIGNATURE / PROVIDER ID
DATE / TIME

\section{References}

1. U.S. Department of Health and Human Services (2008) Surgeon general's call to action to prevent deep vein thrombosis and pulmonary embolism. http://www.surgeongeneral.gov/topics/ deepvein/. Accessed 29 Sept 2008

2. Heit JA, Melton LJ, Lohse CM et al (2001) Incidence of venous thromboembolism in hospitalized patients vs. community residents. Mayo Clin Proc 76:1102-1110

3. Dentali F, Douketis JD, Gianni M, Lim W, Crowther MA (2007) Meta-analysis: anticoagulant prophylaxis to prevent symptomatic venous thromboembolism in hospitalized medical patients. Ann Intern Med 146(4):278-288

4. Heit JA, O'Fallon WM, Petterson TM et al (2002) Relative impact of risk factors for deep vein thrombosis and pulmonary embolism. Arch Intern Med 162:1245-1248

5. Tapson VF, Hyers TM, Waldo AL et al (2005) Antithrombotic therapy practices in US hospitals in an era of practice guidelines. Arch Intern Med 165:1458-1464

6. Clagett GP, Anderson FA, Heit JA et al (1995) Prevention of venous thromboembolism. Chest 108:312-334
7. Geerts WH, Bergqvist D, Pineo GF, Heit JA et al (2008) Prevention of venous thromboembolism: ACCP evidence-based clinical practice guidelines (8th edition). Chest 133(Suppl 6):381S-453S

8. Goldhaber SZ, Tapson VF (2004) A prospective registry of 5, 451 patients with ultrasound-confirmed deep vein thrombosis. Am J Cardiol 93:259-262

9. Monreal M, Kakkar A, Caprini J et al (2004) The outcome after treatment of venous thromboembolism is different in surgical and acutely ill medical patients. Findings from the RIETE registry. $\mathrm{J}$ Thromb Haemost 2:1892-1898

10. Tapson V, Decousus H, Pini M et al (2007) Venous thromboembolism prophylaxis in acutely ill hospitalized medical patients: findings from the International Medical Prevention Registry on Venous Thromboembolism. Chest 132(3):936-945; Epub 2007 June 15

11. Kahn SR, Panju A, Geerts W et al (2007) Multicenter evaluation of the use of venous thromboembolism prophylaxis in acutely ill medical patients in Canada. Thromb Res 119(2):145-155; Epub 2006 Mar 3

12. Cohen AT, Tapson VF, Bergmann JF et al (2008) Venous thromboembolism risk and prophylaxis in the acute hospital care 
setting (ENDORSE study): a multinational cross-sectional study. Lancet 371(9610):387-394

13. NQF (2006) National quality forum endorses consensus standards for prevention and care of venous thromboembolism. Press release. http://216.122.138.39/pdf/news/DVT5-18-06.pdf2006. Accessed 18 May 2006

14. The Joint Commission Manual for Future Performance Improvement Measures (2009) http://www.jointcommission.org/ PerformanceMeasurement/PerformanceMeasurement/Future+ NHQM+Manuals.htm. Accessed 21 July 2009

15. CMS Office of Public Affairs (2009) Fact sheet: CMS proposes additions to list of hospital-acquired conditions for fiscal year 2009. http://www.cms.hhs.gov/apps/media/press/factsheet.asp? Counter=3042. Accessed 10 June 2009

16. Kakkar AK, Davidson BL, Haas SK (2004) Compliance with recommended prophylaxis for venous thromboembolism: improving the use and rate of uptake of clinical practice guidelines. J Thromb Haemost 2:221-227

17. Anderson F, Spencer F (2003) Risk factors for venous thromboembolism. Circulation 107:I-9-I-16

18. Caprini J, Arcelus J, Reyna J (2001) Effective risk stratification of surgical and nonsurgical patients for venous thromboembolic disease. Semin Hematol 38(2 Suppl 5):12-19

19. Gensini GF, Prisco D, Falciani M, Comeglio M, Colella A (1997) Identification of candidates for prevention of venous thromboembolism. Semin Thromb Hemost 23(1):55-67

20. Haas S (2002) Venous thromboembolic risk and its prevention in hospitalized medical patients. Semin Thromb Hemost 28(6):577583

21. Motykie G, Zebala L, Caprini J et al (2000) A guide to venous thromboembolism risk factor assessment. J Thromb Thrombolysis 9:253-262

22. Maynard G, Morris T, Jenkins I, Stone S, Lee J, Renvall M, Fink E, Schoenhaus R (2009) Optimizing prevention of hospitalacquired venous thromboembolism: prospective validation of a VTE risk assessment model. J Hosp Med 4(7). doi:10.1002/ jhm.562

23. Maynard G, Stein J (2009) Preventing hospital-acquired venous thromboembolism - a guide for effective quality improvement. Society of Hospital Medicine website, VTE quality improvement resource room. http://www.hospitalmedicine.org/Resource RoomRedesign/RR_VTE/VTE_Home.cfm. Accessed 14 July 2009

24. Maynard G, Stein J (2008) Preventing hospital-acquired venous thromboembolism: a guide for effective quality improvement, version 3.3. Society of Hospital Medicine supplement. The Hospitalist 12(8): 1-40

25. Maynard G, Stein J (2009) Preventing hospital-acquired venous thromboembolism: a guide for effective quality improvement. Prepared by the Society of Hospital Medicine. AHRQ publication no. 08-0075. Agency for Healthcare Research and Quality, Rockville, MD. September 2008. http://www.ahrq.gov/qual/ vtguide/. Accessed 14 July 2009

26. Shojania KG, McDonald KM, Wachter RM, Owens DK (2004) Closing the quality gap: a critical analysis of quality improvement strategies. Agency for Healthcare Research and Quality, Rockville, MD

27. Kleber F-X, Witt C, Vogel G, Koppenhagen K, Schomaker U, Flosbach CW (2003) Randomized comparison of enoxaparin with unfractionated heparin for the prevention of venous thromboembolism in medical patients with heart failure or severe respiratory disease. Am Heart J 145:614-621

28. Lechler E, Schramm W, Flosbach CW (1996) The venous thrombotic risk in non-surgical patients: epidemiological data and efficacy/safety profile of a low-molecular-weight heparin (enoxaparin). The Prime Study Group. Haemostasis 26(Suppl 2):49-56
29. Colwell CW Jr, Spiro TE, Trowbridge AA et al (1994) Use of enoxaparin, a low-molecular-weight heparin, and unfractionated heparin for the prevention of deep venous thrombosis after elective hip replacement: a clinical trial comparing efficacy and safety. J Bone Joint Surg Am 76:3-14 [Erratum: J Bone Joint Surg Am 1994;76:4741]

30. Geerts WH, Jay RM, Code KI et al (1996) A comparison of lowdose heparin with low-molecular-weight heparin as prophylaxis against venous thromboembolism after major trauma. N Engl J Med 335:701-707

31. Bullock-Palmer RP, Weiss S, Hyman C (2008) Innovative approaches to increase deep vein thrombosis prophylaxis rate resulting in a decrease in hospital-acquired deep vein thrombosis at a tertiary-care teaching hospital. J Hosp Med 3(2):148-155

32. Cohn SL, Adekile A, Mahabir V (2006) Improved use of thromboprophylaxis for deep vein thrombosis following an educational intervention. J Hosp Med 1:331-338

33. Stein J, Chernetsky Tejedor S, Shabbir H, O'Malley E (2008) Situational awareness improves prevalence of VTE prophylaxis on multiple nursing units. J Hosp Med 3(S1):41

34. Schunemann HJ, Cook D, Grimshaw J et al (2004) Antithrombotic and thrombolytic therapy: from evidence to application: the Seventh ACCP Conference on Antithrombotic and Thrombolytic Therapy. Chest 126:688S-696S

35. Tooher R, Middleton P, Pham C et al (2005) A systematic review of strategies to improve prophylaxis for venous thromboembolism in hospitals. Ann Surg 241:397-415

36. Oxman AD, Thomson MA, Davis DA, Haynes RB (1995) No magic bullets: a systematic review of 102 trials of interventions to improve professional practice. CMAJ 153:1423-1431

37. Levi D, Kupfter Y, Seneviratne C, Tessler S (1998) Computerized order entry sets and intensive education improve the rate of prophylaxis for deep vein thrombophlebitis. Chest 114S:280S

38. Dobesh PP, Stacy ZA (2005) Effect of a clinical pharmacy education program on improvement in the quantity and quality of venous thromboembolism prophylaxis for medically ill patients. $\mathbf{J}$ Manag Care Pharm 11:755-762

39. Timmons S, O'Callaghan C, O'Connor M et al (2005) Audit guided action can improve the compliance with thromboembolic prophylaxis prescribing to hospitalized, acutely ill older adults. J Thromb Haemost 3:2112-2113

40. Mosen D, Elliott CG, Egger MJ et al (2004) The effect of a computerized reminder system on the prevention of postoperative venous thromboembolism. Chest 125:1635-1641

41. Kucher N, Koo S, Quiroz R et al (2005) Electronic alerts to prevent venous thromboembolism among hospitalized patients. N Engl J Med 352:969-977

42. Durieux P, Nizard R, Ravaud P, Mounier N, Lepage E (2000) A clinical decision support system for prevention of venous thromboembolism. JAMA 283:2816-2821

43. Dexter PR, Perkins S, Overhage JM, Maharry K, Kohler RB, McDonald CJ (2001) A computerized reminder system to increase the use of preventive care for hospitalized patients. $\mathrm{N}$ Engl J Med 345:965-970

44. Lachiewicz PF (2009) Prevention of symptomatic pulmonary embolism in patients undergoing total hip and knee arthroplasty: clinical guideline of the American Academy of Orthopaedic Surgeons. Instr Course Lect 58:795-804

45. Lyman GH, Khorana AA, Falanga A et al (2007) American Society of Clinical Oncology guideline: recommendations for venous thromboembolism prophylaxis and treatment in patients with cancer. J Clin Oncol 25:5490-5505

46. Committee on Practice Bulletins-Gynecology, American College of Obstetricians and Gynecologists (2007) ACOG practice bulletin no. 84: prevention of deep vein thrombosis and pulmonary embolism. Obstet Gynecol 110(2 pt 1):429-440 